\title{
Application of Brain Stimulation Techniques during Pregnancy
}

\author{
Preeti Sinha ${ }^{1} \odot$, Nishant Goyal ${ }^{2}$, Radhika Kelkar ${ }^{3}$, Vidya Kote Lingappayya ${ }^{4}$
}

\begin{abstract}
Pregnancy is associated with an increased vulnerability to developing psychiatric illnesses that, if left untreated, pose the risk of negative consequences for the mother and the fetus. At the same time, there is a risk of teratogenicity and other possible interference with fetal development associated with pharmacotherapy. Taking into account all these factors, brain stimulation techniques are emerging as reasonable alternatives for the treatment of psychiatric disorders during pregnancy. Here, electroconvulsive therapy (ECT) has the advantage of better evidence of efficacy and faster response. The adverse effects associated with ECT specifically for mother and fetus during pregnancy can be mitigated with proper precautions, monitoring, and multidisciplinary care. Repeated transcranial magnetic stimulation (rTMS) is also emerging to be effective and safe during pregnancy, although the evidence is currently more related to its applicability in depression. This article describes various aspects of ECT and rTMS with the aim of improving their utility as treatment modalities for psychiatric disorders during pregnancy. The other brain stimulation modalities are not much explored for psychiatric disorders during pregnancy and hence are described briefly.

Keywords: Depression, Electroconvulsive therapy, Mental disorders, Pregnancy, Transcranial magnetic stimulation.

Indian Journal of Private Psychiatry (2021): 10.5005/jp-journals-10067-0087
\end{abstract}

\section{INTRODUCTION}

Psychosocial stressors along with biochemical and hormonal changes during pregnancy increase vulnerability to the risk of relapse or recurrence of mental illness and new onset of psychiatric disorders. They predispose the mother as well as the fetus to negative consequences if left untreated. These include poor antenatal and perinatal care, low-birth weight, preterm birth, abortion, and other negative pregnancy outcomes. ${ }^{1-3}$ Depression is one of the common psychiatric clinical entities with a prevalence of $12-36 \%$ during pregnancy. ${ }^{4}$ Related illness, bipolar disorder, also poses a significant risk of relapse in women during and after pregnancy. It has rapid onset of symptoms and is also associated with psychotic characteristics. ${ }^{3,5}$ Schizophrenia, another "severe mental illness," can have exacerbation during this period similar to bipolar disorder.

Similar to other drugs, psychotropics also increase concerns about teratogenic effects, including neurodevelopmental effects and delivery and neonatal complications, and can have pregnancyassociated pharmacodynamic and pharmacokinetic changes. ${ }^{5,6}$ Therefore, pharmacotherapy for the treatment of psychiatric disorders during pregnancy requires careful consideration and risk-benefit analysis on a case-by-case basis before making a treatment decision. Modalities under brain stimulation can be valid treatment alternatives during pregnancy. Although good quality randomized placebo-controlled trials are not available due to ethical concerns for many brain stimulation treatments, empirical evidence is available in the form of case reports, series, reviews, and meta-reviews to support their use during pregnancy. Here, we discuss in detail the electroconvulsive therapy (ECT), a wellestablished and effective treatment with reasonable literature on its utility during pregnancy. This is followed by one of the relatively novel treatments, repeated transcranial magnetic stimulation (rTMS). Other brain stimulation techniques, including transcranial direct current stimulation (tDCS), transcranial alternate current
1,3 Department of Psychiatry, National Institute of Mental Health and Neurosciences, Bengaluru, Karnataka, India

${ }^{2}$ Central Institute of Psychiatry, Ranchi, Jharkhand, India

${ }^{4}$ Department of Geriatric Psychiatry, King George Medical University, Lucknow, Uttar Pradesh, India

Corresponding Author: Preeti Sinha, Department of Psychiatry, National Institute of Mental Health and Neurosciences, Bengaluru, Karnataka, India, Phone: +91 9480829514, e-mail: preetisinha@nimhans.ac.in

How to cite this article: Sinha P, Goyal N, Kelkar R, et al. Application of Brain Stimulation Techniques during Pregnancy. Ind J Priv Psychiatry 2021;15(2):57-61.

Source of support: Nil

Conflict of interest: None

stimulation (tACS), vagal nerve stimulation (VNS), and deep brain stimulation (DBS), have limited evidence of applicability during pregnancy $^{7}$ and are described here briefly.

\section{Electroconvulsive Therapy in Pregnancy}

Electroconvulsive therapy is one of the most effective treatments in psychiatry that has stood the test of time. ${ }^{8}$ However, it is significantly an underused treatment modality due to stigma, concerns about its safety during pregnancy, and lack of access to the treatment facility. ${ }^{9}$ It is important for the readers to know that the American Psychiatric Association and the American College of Obstetricians and Gynecologists jointly have stated that ECT should be considered a safe and effective treatment option for refractory or life-threatening depression during pregnancy. ${ }^{10,11}$ We would now describe here in detail the indications for ECT, the associated adverse events in the mother and baby, and the ways to prevent and manage them during the pregnancy and postpartum period.

(-) The Author(s). 2021 Open Access This article is distributed under the terms of the Creative Commons Attribution 4.0 International License (https://creativecommons. org/licenses/by-nc/4.0/), which permits unrestricted use, distribution, and non-commercial reproduction in any medium, provided you give appropriate credit to the original author(s) and the source, provide a link to the Creative Commons license, and indicate if changes were made. The Creative Commons Public Domain Dedication waiver (http://creativecommons.org/publicdomain/zero/1.0/) applies to the data made available in this article, unless otherwise stated. 


\section{Indications}

Electroconvulsive therapy is a valid treatment modality during pregnancy for many psychiatric conditions, particularly psychiatric emergencies, as it provides a fast and effective response and avoids the potential side effects of pharmacotherapy. These psychiatric conditions include treatment-resistant mood and psychotic disorders, severe depression, high risk of suicide, risk of harm to the baby, catatonia, aggression, psychotic agitation, severe physical decline, and other life-threatening conditions. ${ }^{10}$ The duration of treatment and the response to ECT are similar in pregnant and postpartum patients as in any other individual..$^{12,13}$

\section{Risks to the Mother}

Most adverse events occur in a similar way during pregnancy and the postpartum period as they occurred at other times. However, in the available literature, specific negative outcomes and adverse events for this period are reported as enlisted in various systematic reviews. ${ }^{13-15}$ However, it is important to note that the cause-andeffect relationship is not established for most of these events. Certain complications occurred several weeks after the last ECT session, while some other complications occurred in relation to comorbid medical conditions, such as pneumonia, hypoxia, hypertension, and sickle cell anemia. ${ }^{16}$

The most common adverse events reported were premature contractions and premature labor, which occurred more frequently during the second and third trimesters. Being aware of these complications helped to detect and manage them effectively without serious consequences for the fetus or the neonate. Vaginal bleeding was observed more frequently in the first trimester and was primarily self-limiting without association with spontaneous abortion. ${ }^{15-17}$

\section{Risks to the Fetus}

The most common direct risks to the fetus that were reported are cardiac arrhythmias, bradycardia during the tonic phase, irregular cardiac rhythm postictally, and reduced cardiac variability. No association was confirmed between ECT and any congenital anomaly. $^{14,16}$

\section{Anesthesia-related Issues}

Anesthetic agents used for ECT have rapid induction and fast recovery properties and, hence, are devoid of significant teratogenic effects. Only the usual agents, such as propofol, thiopental, and methohexital, are used. However, these agents easily cross the placental barrier and can cause a slowing of the fetal heart rate and temporary sedation. ${ }^{18,19}$ These risks can be minimized by administering lower doses and monitoring the fetal heart rate throughout the procedure.

In modified ECT, a muscle relaxant is given to minimize the motor component of convulsions. Succinylcholine, the most commonly used muscle relaxant, crosses the placenta but attains negligible levels in the fetal circulation. It is also not associated with significant teratogenic effects. Patients with risk of malignant hyperthermia can be given a nondepolarizing agent, such as rocuronium and atracurium. ${ }^{20}$

Due to the reduced tone of the gastroesophageal sphincter, there is a risk of aspiration in pregnant patients. Precautions, such as stopping anticholinergic agents 24 hours before the session and maintaining strict fasting instructions ( 8 hours for solid foods and 2 hours for liquids), should be followed. In addition, a prophylactic antireflux agent can be given, such as a proton-pump inhibitor and sodium citrate, before the procedure. ${ }^{17,21}$
Hypotension can occur in the third trimester when the patient is in the supine position as the gravid uterus exerts pressure on the inferior vena cava. Therefore, pregnant patients should be placed in the left lateral decubitus position during the procedure. ${ }^{12,15}$ Additional precautionary measures should be taken to reduce the risk of premature labor and uterine contractions. It can become precipitated as a result of long hours of fasting and dehydration. Nonglucose-containing IV fluids can be started before and after ECT. $^{10,12,17}$

\section{Fetal Monitoring}

A common recommendation is to monitor fetal cardiac activity before and after ECT using fetal Doppler. ${ }^{12,14}$ It is recommended that if the fetal heart rate is reduced, consider giving oxygen and ensure that the left lateral decubitus removes the gravid uterus laterally. Some experts also recommend a higher level of monitoring by using external fetal monitoring for several hours before and after ECT and a nonstress test using a tocometer before and after the procedure. This is particularly recommended in cases of high-risk pregnancy and late stages of gestation. ${ }^{21,22}$

\section{Procedural Considerations}

Another safety procedure is to record the maternal echocardiogram and arterial oxygen saturation throughout the procedure. If uterine contractions are suspected, tocodynamometry can be used. Although hyperventilation is routinely practiced before ECT, it is avoided in pregnant patients. It is recommended to practice preoxygenation, but hyperventilation runs a risk of causing fetal hypoxia, as respiratory alkalosis affects oxygen release to the fetal hemoglobin. ${ }^{12,15,17}$

Although there is no difference in general anesthesia techniques used in pregnant patients, inhalational agents may be preferred due to the added advantage of causing tocolysis. ${ }^{19}$ The biphasic hemodynamic response, i.e., parasympathetic overdrive during the tonic phase followed by sympathetic surge during the clonic phase of ECT, should be carefully monitored. Maternal bradycardia can be managed by administering glycopyrrolate $2 \mathrm{mg}$ intravenously, as atropine has a risk of causing fetal distress by crossing the placenta and leading to fetal tachycardia. ${ }^{14,19,20}$

\section{Postprocedure Care}

After the ECT, a thorough assessment is required. If uterine contractions last more than 5 minutes, then they must be suppressed by giving tocolytic agents, such as beta-adrenergic agents (e.g., ritodrine). ${ }^{14}$ Patients should be monitored for symptoms, such as abdominal pain and vaginal bleeding. Abdominal pain can be treated with IV $4 \mathrm{mg}$ of magnesium sulfate diluted in $100 \mathrm{~mL}$ of saline. In case of vaginal bleeding, bed rest withholding additional ECT sessions until the cause of bleeding is identified and dealt with accordingly by liaising with the obstetrician. ${ }^{14}$ Symptoms of nausea and vomiting can be managed with ondansetron, metoclopramide, or prochlorperazine. Post-ECT headache can be treated according to routine management by giving nonsteroidal anti-inflammatory drugs (NSAIDS), such as acetaminophen or paracetamol. ${ }^{17}$

It is advised to closely monitor symptoms, such as cough, fever, and breathing difficulty, and to assess for aspiration pneumonia in such cases. Furthermore, if fetal movements are found to be reduced, it is recommended to consult the obstetrician at the earliest. 


\section{Multidisciplinary Care}

Giving ECT to a pregnant patient is a collaborative decision. Liaison with the obstetrician is an essential step, as it is important to evaluate the patient for risk factors for spontaneous abortion, preterm labor, abruption, and uteroplacental insufficiency before starting ECTs. ${ }^{10,17}$ Pelvic exam to assess vaginal bleeding and vaginal dilatation, fetal assessment by performing nonstress tests, and ultrasound are some other important considerations. ${ }^{12,17}$ In high-risk cases for preterm labor, it is essential to keep the caesarean section tray ready. In such cases, ECT can be administered in the labor room itself. $^{12,17}$ Liaison with the neonatologist is also as important, and both specialists should be aware of the timing of the procedure in order to respond to any possible complications.

\section{rTMS IN PREgNANCY}

Due to its noninvasive nature and minimal adverse effects, rTMS has a potential option to be used in this special population. We would begin with evidence of the efficacy of rTMS in pregnancy in depression and then in other psychiatric disorders.

\section{Efficacy of rTMS in Prenatal Depression}

The United States Food and Drug Administration has approved the use of TMS for patients with major depression, obsessive compulsive disorder (OCD) and migraine in the adult population. Recommendations are not available for the use of TMS in pregnancy. Recently, efforts have been made to generate evidence in the field of neuromodulation during pregnancy, including rTMS. Depression appears to be the most commonly studied entity with controlled and open-label study designs. A randomized controlled trial that compared active $1 \mathrm{~Hz}$ rTMS with right dorsolateral prefrontal cortex (DLPFC) with sham stimulation in 22 women with major depressive disorder in their second or third trimesters of pregnancy. Subjects received 20 sessions of a single train of 900 pulses per session at a $100 \%$ motor threshold. They reported a response rate of $81.82 \%$ for the active coil and $45.45 \%$ for the sham coil $(p=0.088){ }^{23}$

Uncontrolled or open-label studies also report a clinical response ranging from 41.4 to $71.4 \%$ in peripartum depression. ${ }^{24} \mathrm{An}$ uncontrolled study examined 30 pregnant patients with depression who received rTMS on the left prefrontal cortex at $100 \%$ of the motor threshold, $25 \mathrm{~Hz}$ stimulation, a total of 1,000 magnetic pulses, and 18 sessions in 3 weeks. At the end of 3 weeks, $41.4 \%$ of the patients showed a significant improvement with a reduction of more than $50 \%$ in the Hamilton depression rating (HAMD) score. ${ }^{25}$ There are anecdotal reports of pregnant women in their third trimester, suffering from major depression, who responded with intermittent theta-burst stimulation. ${ }^{26,27}$

\section{Evidence in Psychiatric Conditions Other than Depression}

There is a case report of TMS treatment of tokophobia (fear of pregnancy and childbirth) being treated with TMS. The patient received intermittent theta-burst stimulation (600, 3-pulse bursts at $5 \mathrm{~Hz}$, in 2 seconds train and 8 seconds intertrain interval, at $90 \%$ of resting motor threshold) over left DLPFC and continuous thetaburst stimulation (600,3-pulse bursts at $5 \mathrm{~Hz}$, in 2 seconds train at $90 \%$ of resting motor threshold) over right DLPFC. She needed 20 sessions until 28 weeks of gestation and tolerated them well. ${ }^{28}$

There is a report of managing chronic akathisia in a pregnant lady with a single session of $1 \mathrm{~Hz}$ rTMS on the right M1, at $90 \%$ of resting motor threshold (RMT) using a figure-of-eight coil in her 20 weeks of gestation. She had a history of major depressive disorder that achieved remission after a 6-week course of rTMS, experienced worsening of fluoxetine-associated akathisia with pregnancy. ${ }^{29}$ Furthermore, there is a report of pregnant women with psychogenic nonepileptic seizures (PNES) who had been treated with theta-burst stimulation ${ }^{30}$ and reports of treatmentrefractory migraine with aura in three pregnant women who had been treated with single-pulse TMS in the second trimester. ${ }^{31}$

Subsequently, the three patients gave birth without complications to healthy children and continued to treat in the postpartum period.

\section{Safety, Tolerability, and Teratogenicity}

Maternal safety was confirmed in all published efficacy trials, case series, and case reports. Most of the patients received rTMS in the second and third trimesters. The reported adverse effects do not differ significantly from the sham stimulations. Late preterm births were reported in three cases; however, they may not be directly linked to rTMS. $^{23}$ Supine hypotension syndrome characterized by dizziness and low blood pressure occurring 3-10 minutes of lying down, during the rTMS session has been reported in two pregnant women. However, repositioning the patient with a wedge placed under the right lower back resolved the symptom. ${ }^{32}$ Theta-burst stimulation has the potential to overcome this problem as it can be completed in a shorter time per session. ${ }^{27}$

Direct stimulation of the lumbar spine should be avoided in pregnant women according to the recommendations of the consensus conference on safety guidelines on TMS held in 2008 in Italy. ${ }^{33}$ The target site of rTMS in psychiatric disorders should be transcranial, which is less likely to cause any effect on the fetus as the magnetic field attenuates rapidly with distance. ${ }^{33}$

Eryılmaz et al. examined the impact of rTMS on the neurodevelopment of children of 18 mothers who received this treatment in the left prefrontal cortex for 18 days during their second or third trimesters. The study had a control group consisting of children of 26 mothers who had a history of untreated depression during their pregnancy. In addition to perinatal and pregnancyrelated outcomes, early developmental characteristics of the children were evaluated using the Ankara Developmental Screening Inventory. It was concluded that exposure to rTMS during pregnancy is not associated with poorer cognitive or motor development outcomes in children up to $18-62$ months. ${ }^{34}$

The most recent review by Pridmore et al. summarized 10 reports over 20 years, including one controlled trial, and had a total of 67 births. Stimulation was applied in all trimesters, at low and high frequency, both as bilateral stimulation or as intermittent thetaburst stimulation. Mother or fetus did not experience any serious event in any of the reports. Randomized controlled studies with a larger sample size can improve confidence in the use of rTMS during pregnancy. ${ }^{35}$

Yanamadala et al. estimated the maximum peak values of the electric field in the fetal area for every fetal tissue separately for a TMS intensity of one standard motor threshold (SMT) unit using a customized full body finite element model (FEM) of pregnant woman. It was calculated that, when the closest distance between the center of the coil and the uterus is greater than or equal to $60 \mathrm{~cm}$, i.e., $2 \mathrm{ft}$, the maximum peak electric field in the fetal volume including placenta, uterus, and amniotic fluid is expected to be less than or equal to $100 \mathrm{mV} / \mathrm{m}$. This value is significantly lower than the recommended safe limit of $800 \mathrm{mV} / \mathrm{m} .{ }^{36}$ The findings of 
this study illustrate the underlying mechanism of fetal safety of TMS in pregnancy.

\section{Acceptability}

One of the main tenets in the practice of evidence-based medicine is the values and acceptability of the patient. Although the available literature to date has been supportive of the use of rTMS during pregnancy, lack of awareness combined with fear of risk to the fetus can pose a significant challenge in the acceptability of rTMS as an option for the treatment of psychiatrist disorders during the peripartum. In a survey conducted on 1,500 pregnant women in an outpatient unit, $25 \%$ of the participants had depression and TMS was considered an unacceptable treatment option by virtually all women. However, an informational video was shown to all pregnant women about TMS that later improved the acceptability of rTMS as a treatment option. ${ }^{37}$

\section{Other Brain Stimulation Techniques (tDCS, tTACS, VNS, DBS)}

The $t D C S$ has the potential to become a third treatment option for depression during pregnancy. To date, based on available evidence, tDCS is considered a safe, easy-to-handle, and portable brain stimulation technique and has been used to treat depression for more than a decade in numerous clinical trials, yet there is currently an insufficient level of evidence for tDCS during pregnancy. The result of the first pilot RCT published in 2019 examined the efficacy, tolerability, and safety of tDCS treatment in depressed pregnant women and showed particular promise up to 4 weeks of postpartum. ${ }^{38}$ In another recent open-label study with six pregnant women, tDCS was well tolerated without any major fetal or maternal adverse events associated with tDCS. The improvement in depression was also significant. ${ }^{39}$ The current portability of tDCS warrants further trials, as it could potentially be accessed by many more patients. Future studies should pay careful attention to the state of the brain during stimulation.

The tACS could be a potential treatment option for antenatal depression. There is only one published case report that examines the efficacy and the safety of this technique in a depressed pregnant woman, ${ }^{40}$ and proper pilot studies are needed to advance this modality. VNS was long ago approved for long-term adjunctive treatment of chronic or recurrent depression, but in a singlepublished case report, VNS therapy provided effective adjunctive treatment for the patient's depression during pregnancy and delivery; VNS was safe for the patient and her child. ${ }^{7}$ Earlier, an observational study on VNS use in 25 women with epilepsy did not find any evidence of teratogenicity or possible impact on uterus through vagus nerve. ${ }^{41}$ DBS is also researched insufficiently in pregnant women. In psychiatric disorders, it is used in treatment refractory OCD and Tourette syndrome (TS). A retrospective case series of 11 women treated with DBS for OCD, TS, dystonia, or Parkinson's disease reported uneventful pregnancies except spontaneous early abortion of one fetus in a twin pregnancy. ${ }^{42}$

\section{Conclusions and Future Directions}

With the existing literature, brain stimulation treatments in the form of ECT and rTMS appear to cover the limitations associated with psychotropic during pregnancy. The efficacy of ECT for the regular population holds good for pregnant women as well. Recent reviews and the meta-review support the rarity of teratogenic or other medical adverse effects on the fetus or pregnant woman. But this definitely requires specific precautions, monitoring, and additional steps according to the trimester to be followed along with close liaison with an obstetrician. The support from an obstetrician would also be a good strategy in rTMS, although it is generally much safer. Evidence for the use of rTMS during pregnancy is less available than that of ECT. However, rTMS, particularly theta-burst stimulation, has high potential in depression, as well as other psychiatric conditions, such as OCD and resistant auditory hallucinations. Its noninvasive nature and the absence of an anesthetic procedure enhance the possibility of use during pregnancy. Similarly, tDCS and tACS have scope to be utilized during pregnancy but needs research at a greater level. Invasive brain stimulation treatments, VNS and DBS may be helpful in treatment of refractory cases and good RCTs can increase confidence. To conclude, brain stimulation treatments, particularly ECT and rTMS, are underutilized to their potential in this population due to fear of adverse effects and lack of awareness about their utility, respectively. The conduct of largescale prospective multicentric studies would be able to provide evidence of effectiveness, safety, and tolerability during pregnancy.

\section{OrCID}

Preeti Sinha 이 https://orcid.org/0000-0001-5033-7195

\section{References}

1. Fekadu Dadi A, Miller ER, Mwanri L. Antenatal depression and its association with adverse birth outcomes in low and middleincome countries: a systematic review and meta-analysis. PLoS One 2020;15(1):e0227323. DOI: 10.1371/journal.pone.0227323.

2. Doktorchik C, Premji S, Slater D, et al. Patterns of change in anxiety and depression during pregnancy predict preterm birth. J Affect Disord 2018;227:71-78. DOI: 10.1016/j.jad.2017.10.001.

3. Viguera AC, Whitfield T, Baldessarini RJ, et al. Risk of recurrence in women with bipolar disorder during pregnancy: prospective study of mood stabilizer discontinuation. Am J Psychiatry 2007;164(12):18171824; quiz 1923. DOI: 10.1176/appi.ajp.2007.06101639.

4. Bennett HA, Einarson A, Taddio A, et al.Prevalence of depression during pregnancy: systematic review. Obstet Gynecol 2004;103(4):698-709. DOI: 10.1097/01.AOG.0000116689.75396.5f

5. Meador KJ, Baker GA, Browning $\mathrm{N}$, et al. Foetal antiepileptic drug exposure and cognitive outcomes at age 6 years (NEAD study): a prospective observational study. Lancet Neurol 2013;12(3):244-252. DOI: 10.1016/S1474-4422(12)70323-X.

6. Meador KJ, Baker GA, Browning N, et al. Cognitive function at 3 years of age after foetal exposure to antiepileptic drugs. N Engl J Med 2009;360(16):1597-1605. DOI: 10.1056/NEJMoa0803531.

7. Konstantinou GN, Vigod SN, Mehta S, et al. A systematic review of non-invasive neurostimulation for the treatment of depression during pregnancy. J Affect Disord 2020;272:259-268. DOI: 10.1016/j. jad.2020.03.151.

8. Gazdag G, Ungvari GS. Electroconvulsive therapy: 80 years old and still going strong. World J Psychiatry 2019;9(1):1-6. DOI: 10.5498/ wjp.v9.i1.1.

9. Birkenhager TK, Diermen L van. Electroconvulsive therapy: we are hesitant to use the most effective treatment for severe depression. Acta Psychiatr Scand 2020;141(4):301-303. DOI: 10.1111/acps.13171.

10. Jaffe R. The practice of electroconvulsive therapy: recommendations for treatment, training, and privileging: a task force report of the American Psychiatric Association, 2nd ed. Am J Psychiatry 2002;159(2):331. DOI: 10.1176/appi.ajp.159.2.331.

11. ACOG Committee on Practice Bulletins--Obstetrics. ACOG Practice Bulletin: clinical management guidelines for obstetriciangynecologists number 92, April 2008 (replaces practice bulletin number 87, November 2007). Use of psychiatric medications during 
pregnancy and lactation. Obstet Gynecol 2008;111(4):1001-1020. DOI: 10.1097/AOG.0b013e31816fd910.

12. Ward HB, Fromson JA, Cooper JJ, et al. Recommendations for the use of ECT in pregnancy: literature review and proposed clinical protocol. Arch Womens Ment Health 2018;21(6):715-722. DOI: 10.1007/s00737018-0851-0.

13. Leiknes KA, Cooke MJ, Jarosch-von Schweder L, et al. Electroconvulsive therapy during pregnancy: a systematic review of case studies. Arch Womens Ment Health 2015;18(1):1-39. DOI: 10.1007/s00737-0130389-0.

14. Anderson EL, Reti IM. ECT in pregnancy: a review of the literature from 1941 to 2007. Psychosom Med 2009;71(2):235-242. DOI: 10.1097/ PSY.0b013e318190d7ca.

15. Miller LJ. Use of electroconvulsive therapy during pregnancy. Hosp Community Psychiatry 1994;45(5):444-450. DOI: 10.1176/ ps.45.5.444.

16. Sinha P, Goyal P, Andrade C. A meta-review of the safety of electroconvulsive therapy in pregnancy. J ECT 2017;33(2):81-88. DOI: 10.1097/YCT.0000000000000362.

17. Rose S, Dotters-Katz SK, Kuller JA. Electroconvulsive therapy in pregnancy: safety, best practices, and barriers to care. Obstet Gynecol Surv 2020;75(3):199-203. DOI: 10.1097/ OGX.0000000000000763.

18. Herman NL, Li AT, Van Decar TK, et al. Transfer of methohexital across the perfused human placenta. J Clin Anesth 2000;12(1):25-30. DOI: 10.1016/s0952-8180(99)00130-0.

19. Lovas A, Almos PZ, Peto Z, et al. Anesthesia for electroconvulsive therapy in early pregnancy. J ECT 2011;27(4):328-330. DOI: 10.1097/ YCT.0b013e318213cd00.

20. Kadiyala PK, Kadiyala LD. ECT: a new look at an old friend. Curr Opin Anaesthesiol 2018;31(4):453-458. DOI: 10.1097/ ACO. 0000000000000615.

21. O'Reardon JP, Cristancho MA, von Andreae CV, et al. Acute and maintenance electroconvulsive therapy for treatment of severe major depression during the second and third trimesters of pregnancy with infant follow-up to 18 months: case report and review of the literature. J ECT 2011;27(1):e23-e26. DOI: 10.1097/YCT.0b013e3181e63160.

22. Salzbrenner S, Breeden A, Jarvis S, et al. A 48-year-old woman primigravid via in vitro fertilization with severe bipolar depression and preeclampsia treated successfully with electroconvulsive therapy. J ECT 2011;27(1):e1-e3. DOI: 10.1097/YCT.0b013e3181ca4d22.

23. Kim DR, Wang E, McGeehan B, et al. Randomized controlled trial of transcranial magnetic stimulation in pregnant women with major depressive disorder. Brain Stimulat 2019;12(1):96-102. DOI: 10.1016/ j.brs.2018.09.005.

24. Cole J, Bright K, Gagnon L, et al. A systematic review of the safety and effectiveness of repetitive transcranial magnetic stimulation in the treatment of peripartum depression. J Psychiatr Res 2019;115:142-150. DOI: 10.1016/j.jpsychires.2019.05.015.

25. Hızlı Sayar G, Ozten E, Tufan E, et al. Transcranial magnetic stimulation during pregnancy. Arch Womens Ment Health 2014;17(4):311-315. DOI: 10.1007/s00737-013-0397-0.

26. Sylvén SM, Gingnell M, Ramirez A, et al. Transcranial magnetic intermittent theta-burst stimulation for depression in pregnancy - a case series. Brain Stimulat 2020;13(6):1665-1667. DOI: 10.1016/j. brs.2020.09.013.

27. Trevizol AP, Vigod SN, Daskalakis ZJ, et al. Intermittent theta burst stimulation for major depression during pregnancy. Brain Stimulat 2019;12(3):772-774. DOI: 10.1016/j.brs.2019.01.003.
28. Nanjundaswamy MH, Varshney $\mathrm{P}$, Thanki MV, et al. Theta burst stimulation for tokophobia during pregnancy. Brain Stimul 2019;12(5):1322-1324. DOI: 10.1016/j.brs.2019.06.018.

29. Guerrero Solano JL, Molina Pacheco E. Low-frequency rTMS ameliorates akathisia during pregnancy. J Neuropsychiatry Clin Neurosci 2017;29(4):409-410. DOI: 10.1176/appi.neuropsych.17030053.

30. Agarwal R, Garg S, Tikka SK, et al. Successful use of theta burst stimulation (TBS) for treating psychogenic non epileptic seizures (PNES) in a pregnant woman. Asian J Psychiatry 2019;43:121-122. DOI: 10.1016/j.ajp.2019.05.013.

31. Bhola R, Kinsella E, Giffin N, et al. Single-pulse transcranial magnetic stimulation (STMS) for the acute treatment of migraine: evaluation of outcome data for the UK post market pilot program. J Headache Pain 2015;16:535. DOI: 10.1186/s10194-015-0535-3.

32. Kim DR, Wang E. Prevention of supine hypotensive syndrome in pregnant women treated with transcranial magnetic stimulation. Psychiatry Res 2014;218(1-2):247-248. DOI: 10.1016/j. psychres.2014.04.001.

33. Rossi S, Hallett M, Rossini PM, et al. Safety of TMS Consensus Group. Safety, ethical considerations, and application guidelines for the use of transcranial magnetic stimulation in clinical practice and research. Clin Neurophysiol 2009;120(12):2008-2039. DOI: 10.1016/j. clinph.2009.08.016.

34. Eryılmaz G, Sayar GH, Özten E, et al. Follow-up study of children whose mothers were treated with transcranial magnetic stimulation during pregnancy: preliminary results. Neuromodulation 2015;18(4):255260. DOI: $10.1111 /$ ner.12231.

35. Pridmore S, Turnier-Shea Y, Rybak M, et al. Transcranial Magnetic Stimulation (TMS) during pregnancy: a foetal risk factor. Australas Psychiatry 2021;29(2):226-229. DOI: 10.1177/1039856221992636.

36. Yanamadala J, Borwankar R, Makarov S, et al. Estimates of peak electric fields induced by transcranial magnetic stimulation in pregnant women as patients or operators using an FEM full-body model. 2019. In: Makarov S, Horner M, Noetscher G, editors. Brain and human body modeling: computational human modeling at EMBC 2018. Cham (CH): Springer; 2019 [Chapter 3]. Available from: https://www.ncbi.nlm.nih. gov/books/NBK549562/.

37. Kim DR, Sockol L, Barber JP, et al. A survey of patient acceptability of repetitive transcranial magnetic stimulation (TMS) during pregnancy. J Affect Disord 2011;129(1-3):385-390. DOI: 10.1016/ j.jad.2010.08.027.

38. Vigod SN, Murphy KE, Dennis CL, et al. Transcranial direct current stimulation (tDCS) for depression in pregnancy: a pilot randomized controlled trial. Brain Stimul 2019;12(6):1475-1483. DOI: 10.1016/ j.brs.2019.06.019.

39. Kurzeck AK, Dechantsreiter E, Wilkening A, et al. Transcranial direct current stimulation (tDCS) for depression during Pregnancy: results from an open-label pilot study. Brain Sci 2021;11(7):947. DOI: 10.3390/ brainsci11070947.

40. Wilkening A, Kurzeck A, Dechantsreiter E, et al. Transcranial alternating current stimulation for the treatment of major depression during pregnancy. Psychiatry Res 2019;279:399-400. DOI: 10.1016/ j.psychres.2019.06.009.

41. Voinescu PE, Meador KJ. Is neurostimulation through the vagal nerve safe during pregnancy? Epilepsy Res 2017;137:163-164. DOI: 10.1016/ j.eplepsyres.2017.07.008.

42. Scelzo E, Mehrkens JH, Botzel K, et al. Deep brain stimulation during pregnancy and delivery: experience from a Series of "DBS Babies". Front Neurol 2015;6:191. DOI: 10.3389/fneur.2015.00191. 